\title{
Gastroparesis Versus Functional Dyspepsia: Still Running on Emptying
}

\author{
Judy $\mathrm{Nee}^{1} \cdot$ Johanna Iturrino ${ }^{1}$
}

Published online: 6 February 2019

(c) Springer Science+Business Media, LLC, part of Springer Nature 2019

The most common diagnoses of patients complaining of chronic upper abdominal pain, early satiety, postprandial fullness, and nausea, are functional dyspepsia (FD) or gastroparesis (GP). Based on the Rome IV Criteria, to be diagnosed with FD, patients must display one or more of the following symptoms for greater than 3 of the past 6 months: postprandial fullness, epigastric pain or burning, or early satiety, that are unexplained by other structural or organic cause [1]. These symptoms must be of a magnitude such that usual activities are impacted. Alternatively, to be diagnosed with gastroparesis, patients must have objective evidence of delayed gastric emptying (in the absence of mechanical obstruction) combined with upper gastrointestinal symptoms (i.e., early satiety, postprandial fullness, nausea, vomiting, bloating, and/or upper abdominal pain) [2]. Differentiating between these diagnoses influences treatment options such as prokinetics (in the case of gastroparesis) or acid suppression and neuromodulatory therapy (as in functional dyspepsia).

Disease-specific, validated severity questionnaires for FD are numerous. The Nepean Dyspepsia Index, Patient Assessment of Upper Gastrointestinal Severity Index (PAGI-SYM), Dyspepsia Symptom Severity Index (DSSI), and Severity of Dyspepsia Assessment (SODA) are common questionnaires used to group FD research subjects into homogenous populations and to assess symptoms [3]. Yet, due to overlapping symptoms, the diagnosis of FD vs. GP is far from dichotomous. Efforts to distinguish FD from GP by symptoms alone have often failed. For example, in a recent study of 225 gastroparesis patients who completed Rome IV Diagnostic questionnaire, 90.8\% fulfilled functional dyspepsia criteria (88.3\% with postprandial distress and $59.8 \%$ with epigastric pain syndrome) [4].

Judy Nee

jnee@bidmc.harvard.edu

1 Division of Gastroenterology, Department of Medicine, Beth Israel Deaconess Medical Center, Harvard Medical School, Boston, MA, USA
In this issue of Digestive Diseases and Sciences, Lacey et al. [5] evaluated the potential use of the Gastroparesis Cardinal Symptom Index (GCSI), a questionnaire commonly used in gastroparesis trials, to assess symptom severity in FD patients. The GCSI is a subset of 9 questions derived from the more comprehensive PAGI-SYM, originally developed from focus groups and individual interviews with patients complaining of reflux, FD, and GP. It consists of questions assessing 3 main symptoms: bloating ( 2 questions), nausea (3 questions), and fullness/early satiety (4 questions). Each question assesses the severity of symptoms occurring in the previous 2 weeks from 'none' (0) to 'very severe' (5) with a total score calculated from the average of the 3 main symptoms, with higher GCSI scores indicating more severe symptoms. A 2004 study by Revicki et al. [6] addressing the reliability and validity of the GCSI was performed with 169 patients diagnosed with gastroparesis and 760 with dyspepsia. Mean GCSI scores, which corresponded to clinicianrated symptoms, could be used to differentiate between mild, moderate, and severe symptoms. Not only were the mean GCSI scores significantly higher in GP compared with FD subjects, but all three subscores of bloating, nausea/vomiting, and postprandial fullness were also significantly higher in GP compared to FD.

In the current study, Lacy et al. mailed questionnaires that included the GCSI to 254 FD patients diagnosed at a tertiary care referral center. One hundred and twenty-three patients, predominantly women $(75 \%)$, were ultimately included in the study. All patients fulfilled the Rome III criteria for FD and had undergone an upper endoscopy and gastric emptying scans to exclude organic disease and gastroparesis. Less than half were categorized as postprandial distress syndrome (PDS), followed by $30 \%$ epigastric pain syndrome (EPS) and 20\% mixed PDS-EPS. PDS is characterized postprandial distress and early satiety, while EPS is characterized by epigastric pain or burning. The most bothersome symptom in the FD patients was abdominal pain (33\%) followed by bloating (17\%) and upper abdominal discomfort (15\%). As a comparator, the authors used historical GP controls from the validation study by Revicki et al. in 2004 [6]. Not 
surprisingly, the authors were unable to distinguish FD from GP based on the GCSI questionnaire, though GCSI scores were slightly lower than the historical control gastroparesis patients. Furthermore, the highest subscore in the FD cohort was bloating, followed by postprandial fullness and nausea/vomiting. As in prior trials, higher GCSI scores were correlated with increased FD severity and higher Hospital Anxiety and Depression Scale scores. Nonetheless, the total GCSI score did not correlate with gastric emptying fraction measured at $4 \mathrm{~h}$. Furthermore, when the FD patients were subcategorized into epigastric pain (EP), postprandial distress (PPD), and mixed (both EP and PPD), GCSI scores were also similar, indicating that the GCSI lacked sufficient specificity to distinguish FD subtypes. Interestingly, subjects with self-reported "mild" symptoms reported the highest GCSI scores, greater than those who self-reported "moderate" or "severe" symptoms, driven by the high bloating subscores.

The 2004 study by Revicki et al., from which the historical gastroparesis controls were taken, did report a significant difference in GCSI scores between FD and GP, possibly due to differences in the criteria used by these studies to define FD and GP. The GP cohort used in the 2004 study was diagnosed using a "gastroparesis diagnosis in the medical record (i.e., gastroparesis, gastric motility disorder, delayed gastric emptying)" although gastric emptying times were not reported and were noted to be performed at variable times in relation to questionnaires; additionally, the criteria used to identify FD subjects used in the Revicki study was not described. The current article uses Rome III criteria as assessed by an expert in FGID for the diagnosis of the FD subjects. They report that $26 \%$ of the FD patients had delayed, $4 \%$ had rapid, and $68 \%$ had normal gastric emptying fractions. Since the presence of delayed gastric emptying alone does not equate to gastroparesis, FD subjects in the 2004 study may have been miscategorized as GP subjects, especially those with mildly delayed gastric emptying fractions. It is possible that the similarity in the GCSI scores between FD and GP in this study may have been due to differences in the criteria used to define FD in the current study and GP in the historical control.

The difficult truth is that since these upper GI symptoms are common to both FD and GP, as highlighted by the study by Lacy et al., the GCSI is not useful for making the correct diagnosis. What can help distinguish the two? Prior studies have shown that neither severity nor quality of upper abdominal symptoms correlate sufficiently with delayed gastric emptying. Interestingly, and in contrast to other studies, GCSI subscores for fullness and bloating were associated with gastric emptying fraction measured at $2 \mathrm{~h}$ but not $4 \mathrm{~h}$. The American College of Gastroenterology (ACG) currently advises against the routine use of motility studies, such as gastric emptying scintigraphy, in the evaluation and management of FD. Nevertheless, the ACG guidelines for GP recommend gastric emptying evaluation in those patients with predominant symptoms of nausea and vomiting [7]. In two studies of FD patients, delayed gastric emptying of solids or of liquids was associated with severe vomiting [8, 9]. We would argue that measurement of the gastric emptying fraction for patients on all parts of the spectrum (rapid, normal, mildly delayed, or severely delayed) would appropriately identify those on the opposite sides of the spectrum (normal gastric emptying vs. severely delayed emptying). If patients indeed have severely delayed gastric emptying with nausea and vomiting symptoms in addition to marked weight loss and nutritional deficiencies, a diagnosis of gastroparesis may be appropriate.

Questionnaires based on symptoms alone are not able to exclude alternative diagnoses. Other than FD, other FGIDs such as rumination, chronic idiopathic nausea, chronic idiopathic vomiting, eating disorders, and other related ailments can also have symptoms overlapping with gastroparesis and should be excluded. Perhaps adding upper abdominal pain to questionnaires may help distinguish FD and GP. Still, pain is the predominant symptom in approximately $20 \%$ of these patients and is associated with idiopathic etiology, lack of infectious prodrome, and opiate use [10]. In a study of GCSI-daily diary (DD), which is a daily questionnaire with both GSCI and upper abdominal pain, no change was seen in the abdominal pain score with treatment of gastroparesis [11]. Upper abdominal pain may indicate visceral hypersensitivity and hence help confirm an FD diagnosis, and may have promise as a marker useful for disease monitoring. Yet, a questionnaire such as the GCSI-DD may not be useful in identifying FD patients with or without delayed gastric emptying.

In summary, many patients with gastroparesis and FD share similar symptoms. To distinguish these entities and exclude alternative diagnoses, a thorough history and physical examination should be performed. Patients with alarm features (weight loss) or persistent severe symptoms (particularly vomiting) may require additional diagnostic testing. Although the contribution of gastric emptying measurement to clinical practice remains unclear, in some patients the results may help direct therapy. For example, a patient with mildly delayed gastric emptying, with no evidence of weight loss and who is not underweight, would preferably be diagnosed with functional dyspepsia in order to avoid causing more harm due to more aggressive medical treatment and potentially more invasive procedures that are frequently employed in patients diagnosed with GP. Questionnaires such as the GCSI or the GCSI-DD will continue to be useful for research studies and potentially in practice to assess symptom severity, but they are unlikely to help distinguish these patient populations by their current definitions. 


\section{References}

1. Drossman DA. Functional gastrointestinal disorders: History, pathophysiology, clinical features and Rome IV. Gastroenterology. 2016;150:1262-1279.

2. Camilleri M, Parkman HP, Shafi MA, et al. Clinical guideline: Management of gastroparesis. Am J Gastroenterol. 2013;108:1837; quiz 38.

3. Ang D, Talley NJ, Simren M, et al. Review article: Endpoints used in functional dyspepsia drug therapy trials. Aliment Pharmacol Ther. 2011;33:634-649.

4. Jehangir A, Parkman HP. Rome IV Diagnostic questionnaire complements patient assessment of gastrointestinal symptoms for patients with gastroparesis symptoms. Dig Dis Sci. 2018;63:22312243. https://doi.org/10.1007/s10620-018-5125-1.

5. Lacy BE, Everhart K, Crowell MD. Functional dyspepsia: Clinical symptoms, psychological findings, and GCSI scores. Dig Dis Sci. (Epub ahead of print). https://doi.org/10.1007/s1062 0-018-5347-2.

6. Revicki DA, Rentz AM, Dubois D, et al. Gastroparesis cardinal symptom index (GCSI): Development and validation of a patient reported assessment of severity of gastroparesis symptoms. Qual Life Res. 2004;13:833-844.

7. Moayyedi PM, Lacy BE, Andrews CN, et al. ACG and CAG clinical guideline: Management of dyspepsia. Am J Gastroenterol. 2017;112:988-1013.

8. Sarnelli G, Caenepeel P, Geypens B, et al. Symptoms associated with impaired gastric emptying of solids and liquids in functional dyspepsia. Am J Gastroenterol. 2003;98:783-788.

9. Stanghellini V, Tosetti C, Paternico A, et al. Risk indicators of delayed gastric emptying of solids in patients with functional dyspepsia. Gastroenterology. 1996;110:1036-1042.

10. Hasler WL, Wilson LA, Parkman HP, et al. Factors related to abdominal pain in gastroparesis: Contrast to patients with predominant nausea and vomiting. Neurogastroenterol Motil. 2013;25:e300-e301.

11. Revicki DA, Camilleri M, Kuo B, et al. Evaluating symptom outcomes in gastroparesis clinical trials: Validity and responsiveness of the gastroparesis cardinal symptom index-daily diary (GCSIDD). Neurogastroenterol Motil. 2012;24:e215-e216. 